\title{
Guest-editorial
}

\section{Special issue: Recent developments in hybrid intelligent systems}

\author{
Kaori Yoshida ${ }^{\mathrm{a}}$ and Witold Pedrycz ${ }^{\mathrm{b}}$ \\ ${ }^{a}$ Department of Artificial Intelligence, Kyushu Institute of Technology, Japan \\ E-mail: kaori@ai.kyutech.ac.jp \\ ${ }^{\mathrm{b}}$ Department of Electrical and Computer Engineering, University of Alberta, Canada \\ E-mail: pedrycz@ee.ualberta.ca
}

The human-centric facet of intelligent systems, which are inherently based upon the principle of hybridization of the individual information technologies, is definitely an interesting and highly promising avenue to follow. The diversity of communication channels between information systems and the environment is immense, and their effective realization does call for a truly heterogeneous nature of the underlying technologies and algorithmic frameworks. Consider, for instance, processing visual information, which became omnipresent in many applications including a broad range of inspection and manufacturing systems. Computer vision offered remarkable opportunities of processing therein. Fuzzy sets support a granular view at images and form a setting for their interpretation at the higher, more abstract level. Neural networks and evolutionary optimization provide badly needed learning abilities. The technologies mentioned so far, when combined into an inherently hybrid system, help address the needs of intelligent vision systems and build a coherent and versatile implementation. Managing knowledge at various levels, starting from the detailed pixel-level and moving to the far more general abstract level, becomes crucial to the success of such systems.

This special issue is a testimony to the ongoing innovation and recent developments in hybrid information systems. The study by Shahram Jafari and Ray Jarvis focuses on implementing perception in the context of robotic eye-to-hand coordination. The authors show an integration of different novel concepts to perform scene analysis, hand-eye coordination and object manipulation for robotic tasks. The underlying architectural considerations rely on the usage of the neuro-fuzzy systems in the formation of scene analysis and object recognition. Next, Ghosh and Petkov, in their study, deal with the cognitive evaluation of contour based shape descriptors in image processing. In the sequel, first-order logical networks discussed by Kijsirikul and Lerdlamnaochai offer an interesting insight into the problems of logic-based neurocomputing. The proposed method, called First-Order Logical Neural Network (FOLNN), leads to hybrid structures that combine standard feed-forward neural networks and mechanisms of inductive learning. The study by Sehgal, Gondal, and Dooley is focused on an important and timely issue of handling multiple missing values of genetic expression in problems of analysis and classification of microarray data. The authors offer a mechanism of a k-ranked covariance-based missing value imputation and demonstrate its superiority over the k-nearest neighbor method. 
We are grateful to the Editors-in-chief for their continuous encouragement and support. Our sincere thanks go to the reviewers who were able on a timely basis to offer numerous thorough and constructive comments. Yes, we definitely enjoyed working on this special issue and do hope that it will offer a forum for stimulating discussions fostering further progress in the area.

Kaori Yoshida and Witold Pedrycz

Guest-editors

October 12, 2005 